\title{
Interaction of LHBs with C53 promotes hepatocyte mitotic entry: A novel mechanism for $\mathrm{HBV}$-induced hepatocellular carcinoma
}

\author{
YONGHONG LEI ${ }^{2 *}$, HAO LIU ${ }^{3 *}$, YUYING YANG $^{1}$, XIAOJIE WANG $^{1}$, NA REN $^{1}$, BEN LI $^{1}$, \\ SHUNAI LIU ${ }^{1}$, JUN CHENG ${ }^{1}$, XIAOBING FU ${ }^{2}$ and JINQIAN ZHANG ${ }^{1}$
}

${ }^{1}$ Institute of Infectious Diseases, Beijing Ditan Hospital, Capital Medical University, Beijing 100015; ${ }^{2}$ Wound Healing and Cell Biology Laboratory, The First Affiliated Hospital, General Hospital of PLA, Beijing 100853; ${ }^{3}$ Department of General

Surgery, The School of Traditional Chinese Medicine, Southern Medical University, Guangzhou 510515, P.R. China

Received July 13, 2011; Accepted September 2, 2011

DOI: $10.3892 / o r .2011 .1489$

\begin{abstract}
The pre-S mutant LHBs, especially the pre-S2 type, is believed to be crucial in HBV-associated hepatocellular carcinogenesis. However, the mechanism of HBV-induced hepatocellular carcinoma is not fully understood. To identify the mechanism, pre-S2 LHBs-interacting proteins were studied, by performing a yeast two-hybrid screen of a human liver cDNA library. Screening of the library resulted in the isolation of several positive clones. Sequencing of the positive clones identified the full-length cDNA of the C53 gene. After identification of the interaction, the roles of LHBs on Cdk1, Chk1 activation and mitotic entry were studied. Screening of the library resulted in the isolation of several positive clones, that encoded the full-length cDNA of the C53 gene. We found that C53 interacts with pre-S2 LHBs both in vitro and in vivo, but not with LHBs or other mutants. The binding of pre-S2 LHBs with C53 causes increased Cdk1 activation and mitotic entry, and the function of Chk1 is partially inhibited by the binding of pre-S2 LHBs with C53. Taken together, our results strongly suggest that the binding of pre-S2 LHBs with C53 is a novel negative regulator of the checkpoint response. By counteracting C53, pre-S2 LHBs promotes Cdk1 activation and mitotic entry in unperturbed cell cycle progression and delays the function of Chk1, which may be a novel potential mechanism for HBV-induced hepatocellular carcinoma (HCC).
\end{abstract}

Correspondence to: Dr Jinqian Zhang, Institute of Infectious Diseases, Beijing Ditan Hospital, Capital Medical University, 8 Jingshun East St., Beijing 100015, P.R. China

E-mail: jingwanghou@yahoo.com.cn

Professor Xiaobing Fu, Wound Healing and Cell Biology Laboratory Institute of Basic Medical Science, The First Affiliated Hospital, General Hospital of PLA, Trauma Center of Postgraduate Medical College, 28 FuXing Road, Beijing 100853, P.R. China

E-mail: fuxb@cgw.net.cn; fuxiaobing@vip.sina.com

${ }^{*}$ Contributed equally

Key words: pre-S2 LHBs, C53, interaction, mitotic entry, hepatocellular carcinoma

\section{Introduction}

Hepatitis B virus (HBV) is an enveloped DNA virus that is a common infectious agent worldwide (1). More than 2 billion people have been infected with hepatitis B virus (HBV) and $\sim 350$ million remain chronically infected (2). Most infected patients are able to mount an effective immune response and clear the virus. However, a substantial minority fails to do so and becomes chronically infected. They are susceptible to developing chronic hepatitis, cirrhosis and especially hepatocellular carcinoma $(3,4)$. About $1 / 3$ of the world's population have had contact with $\mathrm{HBV}$, and $\sim 2 / 3$ of all liver cancers are attributable to an HBV infection (5). The human hepatitis B virus (HBV) is a remarkably small, enveloped, DNA-containing pararetrovirus (6). The envelope of HBV is formed by three different surface proteins (HBs) termed L- (large), M- (middle) and S- (small), with a ratio of $\sim 1: 1: 4$ (7). On biogenesis, the HBV $\mathrm{L}$ protein, together with the structurally closely related middle $\mathrm{M}$ and small $\mathrm{S}$ envelope proteins, is expressed from a single ORF of the viral genome by differential translation initiation. As a consequence, the entire sequence of $\mathrm{S}$ is repeated at the $\mathrm{C}$ terminus of $\mathrm{M}$ and $\mathrm{L}$, which contain the additional pre-S2 domain or pre-S2 and pre-S1 domains, respectively (8).

In the late 1990s, two major types of pre-S deletion mutant LHBs were identified and were associated with HCC $(9,10)$. In the two types of pre-S mutant LHBs, pre-S1 and pre-S2 mutant LHBs, the pre-S1 and -S2 regions, respectively, are partially deleted $(11,12)$. They accumulate in the endoplasmic reticulum (ER) and induce strong ER stress (13). Through an ER stressmediated pathway, they cause oxidative stress and DNA damage (14). Through an ER stress-independent pathway, however, pre-S2-mutant LHBs contributes to the increased proliferation of hepatocytes (15). Pre-S2 mutant large HBsAg expressed in hepatocytes cluster into groups and exhibit clonal expansion and growth advantage. Based on the findings of these previous studies, pre-S mutant LHBs, especially the pre-S2 type, is believed to be crucial in HBV-associated hepatocellular carcinogenesis. We previously demonstrated that the pre-S2 LHBs interacted with C53 with yeast two-hybrid analysis. The interaction was further studied.

Jiang et al originally reported that by counteracting Chk1 and antagonizing its function, C53 promotes Cdk1 activation 
and mitotic entry in both the unperturbed cell cycle progression and in the DNA damage response (16). Herein, the interaction between $\mathrm{C} 53$ and pre-S2 LHBs, and the roles of this interaction on Cdk1, Chk1 activation and mitotic entry were studied.

\section{Materials and methods}

Culture cells. L02, OSG-7701 and HL-7702 cells were cultured in Dulbecco's modified Eagle's medium (DMEM) supplemented with $10 \%$ fetal bovine serum and antibiotics. The results obtained from OSG-7701 and HL-7702 cells are not mentioned.

Plasmids. For the yeast two-hybrid assay, the bait plasmid pGBKT7-pre-S2 LHBs was generated by inserting a PCRamplified cDNA fragment containing pre-S2 LHBs into pGBKT7 (Clontech) and pBIND, pEGFP-C1 (Clontech), pGEX-2T vector. Human full-length C53 cDNA and the cDNAs for its truncated mutants (C53N and C53C1-3) were PCR-amplified and subcloned in-frame into pCMV-5a (Sigma). C53 was cloned into pACT, pDsRed1-N1 (Clontech), pCDNA3.1(-) myc/his (A) vector. To construct C53 shRNA expression vectors, we ligated shRNA oligonucleotide template into linearized pAdTrack-CMV vector to generate pAdTrack-CMVC53 expressing C53 shRNA. Oligonucleotide templates were designed and synthesized according to guidelines provided by Clontech, which were described in Jiang et al (17).

All plasmids were verified by restriction enzyme analysis and DNA sequencing. Informed written consent was obtained from the patient. The study was approved by the Ethics Committee according to the Declaration of Helsinki.

Primers. For the yeast two-hybrid assay, the bait plasmid pGBKT7-pre-S2 LHBs was generated by inserting a PCR-amplified cDNA fragment containing pre-S2 LHBS into pGBKT7 (Clontech) using the upstream primer 5'-GCTAGCTATGGAGGACCAT CAGCACGT-3' and the downstream primer 5'-GGATCC AATGTATACCCAAAGAC-3'. Pre-S2 LHBs was cloned into parent vector $\mathrm{pBIND}$ using the forward primer 5'-GGA TCCAAATGGGAGGTTG-3') and the reverse primer 5'-AAGCTTTCAAATGTATACCCAAAG-3'.

Enhanced green fluorescent protein (GFP)-tagged fusion protein constructs were made by inserting PCR-amplified pre-S2 LHBs cDNA fragments into pEGFP-C1 (Clontech) using the upstream primer 5'-AAGCTTATGGGAGGTTGG-3' and the downstream primer 5'-GGATCCAATGTATACCCA AAGAC-3'. Pre-S2 LHBs was cloned into pGEX-2T using the forward primer 5'-GGATCCAAATGGGAGGTTG-3' and the reverse primer 5'-GAATTCAATGTATACCCAAAGAC-3'

Human full-length C53 cDNA and the cDNAs for its truncated mutants (C53N and C53C1-3) were PCR-amplified and subcloned in-frame into pCMV-5a (Sigma), and the resulting constructs were used for expression of C-terminal Flag-tagged C53 in mammalian cells. Primers for full length C53 were 5'-GCGTCGACATGGAGGACCATCAGCAC-3' (forward) and 5'-GCGGTACCCAGAGAGGTTCCCAG-3' (reverse). Primers for C53 C1, C2 and C3 were 5'-GCGTCGACATGTGG GGCGGACTTTGGGGTAG-3' (C1, forward), 5'-GCGTCGA CATGTCTGGCATCTCTGCCGAGG-3' (C2, forward), and 5'-GCGTCGACATGGATGCTGTTGCTTTGCAGAT-3' (C3, forward), respectively. The reverse primer for $\mathrm{C} 53 \mathrm{~N}$ was 5'-CCGGATCCTTCAATCGCATCTTCTGCCAC-3'. C53 was cloned into pACT using the forward primer 5'-GTCGAC TAATGGAGGACCATCAGCACGT-3' and the reverse primer 5'-GGTACCTCACAGAGAGGTTCCCATCA-3'. Using the upstream primer 5'-GCTAGCTATGGAGGACCATCAGCA CGT-3' and the downstream primer 5'-GGTACCATTCACA GAGAGGTTCCCATCA-3', enhanced red fluorescent protein (RFP; 1)- tagged fusion protein constructs were made by inserting PCR-amplified C53 cDNA fragments into pDsRed1$\mathrm{N} 1$ (Clontech). C53 was cloned into pCDNA-3.1(-) myc/his (A) vector using the forward primer 5'-TATCTGATGGAGGAC CATCAGCACGT-3' and the reverse primer 5'-GGTACCAT TCACAGAGAGGTTCCCATCA-3'.

Mutation of LHBs. LHBs was amplified from serum of a patient with chronic hepatitis B (subtype B) in China, using the upstream primer 5'-CGGAATTCCGATGGGAGGTTG-3' and the downstream primer 5'-CCAAGCTTGGTCAAATGTATACCC-3'. Then LHBs cDNA was cloned into pCDNA-3.1(-) myc/his (A) vector.

Site-directed mutagenesis was performed using the Transformer $^{\mathrm{TM}}$ Site-Directed Mutagenesis kit (Clontech) as recommended by the manufacturer. For simplification, amino acid numbering used herein will refer to consecutive numbering of the $\mathrm{L}$ protein rather than to the envelope protein domains (data not shown).

Theselectionprimerwas5'-CAAGTGTATCATAGGCCAA GTACGCCC-3' [nucleotides (nts) 31-57]. The N15S mutation was introduced with the mutagenic primer 5'-GGCATGGGG ACAAGTCTTTCTGTTCCC-3' (nts 31-57). The N123S mutation was introduced with the mutagenic primer 5'-GCCATGCA GTGGAGCTCCACCACATTC-3' (nts 355-381). The N177S mutation was introduced with the mutagenic primer 5'-CGAA CATGGAGAGCACAACATCAGG-3' (nts 518-542). The $\mathrm{N} 320 \mathrm{~K}$ mutation was introduced with the mutagenic primer 5'-CTTCGGACGGAAAGTGCATTGTATTC-3' (nts 947-973).

The resulting product was cut with $\mathrm{SacI}$ used to substitute for the wild-type fragment of pEGFP-C1-LHBs. The fragment containing the N15S, N123S (pre-S2), N177S and N320K mutation were subcloned into the pCDNA-3.1(-) and pGBKT7 vector.

Yeast two-hybrid assay. The bait plasmid pGBKT7-pre-S2 LHBs was used to screen a human liver cDNA library in pGADT7 according to the manufacturer's instructions (Clontech). Transformants were plated on synthetic medium lacking tryptophan, leucine, adenine and histidine but containing $1 \mathrm{mM} 3$-aminotriazole. Approximately 4 million transformants were screened. The screened positive clones were also verified by one-on-one transformations and selection on agar plates lacking tryptophan and leucine, or adenine, histidine, tryptophan and leucine, respectively, and were also processed by a $\beta$-galactosidase assay.

Co-immunoprecipitation. For transfection-based co-immunoprecipitation assays, cells were transfected with the indicated plasmids using Lipofectamine ${ }^{\mathrm{TM}} 2000$ (Invitrogen, Carlsbad, CA, USA), lysed in $0.5 \mathrm{ml}$ lysis buffer (50 mM Tris at $\mathrm{pH} 8.0$, $150 \mathrm{mM} \mathrm{NaCl}, 0.25 \% \mathrm{NP}-40,1 \mathrm{mM}$ DTT and protease inhibitor tablets from Roche), and immunoprecipitated with Protein 
G Plus/Protein A Agarose Suspension beads (Calbiochem) for $3 \mathrm{~h}$ at $4^{\circ} \mathrm{C}$. The beads were washed four times with the lysis buffer, and eluted in SDS sample buffer. The eluted proteins were separated by SDS-PAGE, followed by Western blotting with antibody.

Mammalian two-hybrid analysis. To test the hypothesis of C53 interacting with pre-S2 LHBs in vivo using the CheckMate ${ }^{\mathrm{TM}}$ mammalian two-hybrid system (Promega), plasmids pACTC53 and plasmids pBIND-pre-S2 LHBs were constructed, which were used for co-transfections of cells in culture which were grown on culture in 6-well plates. pACT-C53 plasmid $(2 \mu \mathrm{g})$ and $2 \mu \mathrm{g}$ pBIND-pre-S2 LHBs plasmid were used for co-transfections with Lipofectamine 2000. The plasmids pACTC53+pBIND, pACT+pBIND-pre-S2 LHBs, pACT, pBIND and blank were used for transfections respectively as a negative control. The plasmids pBIND-Id and pACT-MyoD Control Vector were co-transfected as a positive control. At $48 \mathrm{~h}$ after transfection, cells were washed three times with PBS and lysed by passive buffer. Firely luciferase assays were performed using the Dual-Glo Luciferase Assay System (Promega) following the manufacturer's instructions. The firefly results were corrected for transfection efficiency using the Renilla luciferase. Significance was determined using the paired Student's t-test on the mean of three different experiments.

GST pull-down assay. The GST- and His-tagged fusion proteins were expressed and purified by glutathione-sepharose $4 \mathrm{~B}$ beads (GE) and Ni-NTA agarose (GE), respectively. The expression plasmid for C53 was used for in vitro transcription and translation in the TNT system (Promega). The C53 or the purified His-tagged fusion protein was incubated with GST fusion protein bound to glutathione-sepharose beads in $0.5 \mathrm{ml}$ of the binding buffer (50 mM Tris- $\mathrm{HCl}, \mathrm{pH} 7.5,150 \mathrm{mM} \mathrm{NaCl}, 1 \mathrm{mM}$ EDTA, $0.3 \mathrm{mM}$ DTT, $0.1 \% \mathrm{NP}-40$ ) at $4^{\circ} \mathrm{C}$. The beads were precipitated, washed four times with the binding buffer, eluted by boiling in SDS sample buffer, and analyzed by SDS-PAGE. Western blot analysis was performed using anti-His (Santa Cruz). A quantitative measurement of the band intensity was performed using the GE Typhoon Trio (GE, USA).

Co-localization. Cells were grown on glass coverslips in culture plates. Cells were co-transfected with plasmids $2 \mu \mathrm{g}$ of pEGFP-C1-pre-S2 LHBs (green fluorescent protein, GFP) and $2 \mu \mathrm{g}$ pDS-RED1-N1-C53 by Lipofectamine 2000 for $48 \mathrm{~h}$, and then cells were treated with $4 \%$ paraformaldehyde (PFA) for 10 min, washed 3 times with PBS, stained with PBS including $0.1 \mu \mathrm{g} / \mathrm{ml} \mathrm{4}, 6$-diamidino-2-phenylindole (DAPI) for $30 \mathrm{~min}$ at $30^{\circ} \mathrm{C}$. Confocal images were acquired using the Zeiss 510 META confocal microscope.

Retrovirus transduction and C53 knockdown by shRNA. The short hairpin RNA (shRNA) interference oligonucleotides against C53 described previously were inserted into the shRNA shuttle expression vector pAdTrack-CMV. The resultant plasmid was linearized by $P m e I$ and subsequently co-transformed into $E$. coli BJ5183 with an adenoviral backbone plasmid (pAdEasy-1). Subsequently, the recombinant adenoviral plasmid (pAd-C53) was transfected into HEK 293 cells by Lipofectamine 2000 for amplification. Adenovirus was purified by centrifugation in a cesium chloride gradient. Final virus yields were $10^{10}-10^{11} \mathrm{PFU} / \mathrm{ml}$. The Ad-eGFP was constructed similarly using the Ad virus as control. Cells were infected, and the infection efficiency (95-100\%) was determined by GFP expression $24 \mathrm{~h}$ after infection. Cells were harvested for preparation of protein extracts $72 \mathrm{~h}$ after infection.

Antibodies and immunoblotting. Immunoblotting was performed as previously described (18). The following antibodies obtained from Santa Cruz Biotechnology (Santa Cruz, CA, USA) unless otherwise noted, were used at the indicated dilutions for immunoblotting: C53 antibody (1:1,000 dilution), $\beta$-actin $(1: 1,000)$, GST $(1: 1,000)$, Flag $(1: 1,000)$, His $(1: 1,000)$, Cdk1 and p-Y15-Cdk1 (Cell Signaling, 1:1,000), Chk1 (1:2,000), p-S10-Histone H3 (Upstate, 1:3,000), Myc (1:1,000). All affinitypurified and species-specific fluorophore-conjugated secondary antibodies were obtained from Santa Cruz Biotechnology, and used at dilutions between 1:500 and 1:800.

Cell synchronization, BrdU labeling and mitotic index. To avoid potential carry-over effects of plasmids or transfectioninduced cell cycle defects in the previous cycle on the following mitotic entry during the next cycle, we transfected plasmids into cells during the interval between two thymidine blocks, so that we were able to evaluate the direct impact of the interaction of C53 with pre-S2 LHBs on mitotic entry (19). Cells were synchronized by double thymidine block. Briefly, cells were plated at $40 \%$ confluency and arrested with $2 \mathrm{mM}$ thymidine. After $19 \mathrm{~h}$ incubation, cells were washed 4 times with fresh medium and transfected with shRNA vectors (C53 and control) using Lipofectamine 2000. After incubation with DNA-lipid mixture for $3 \mathrm{~h}$, cells were washed twice and incubated in fresh medium for an additional $5 \mathrm{~h}$. Subsequently, cells were cultured in medium containing $2 \mathrm{mM}$ thymidine and $2 \mu \mathrm{g} / \mathrm{ml}$ puromycin for the second arrest and drug selection. After $16 \mathrm{~h}$ incubation, cells were released into the cell cycle by incubation in fresh medium. Cells were collected or fixed at indicated time points and subjected to specific analyses.

BrdU labeling was used to evaluate DNA synthesis. After release from the second thymidine arrest at indicated time points, cells grown in 12-well plates were pulse labeled with BrdU $(50 \mu \mathrm{M})$ for $30 \mathrm{~min}$. After three washes of PBS, cells were fixed with $1 \mathrm{ml}$ of Carnoy's fixative (3 parts methanol, 1 part glacial acetic acid) at $-20^{\circ} \mathrm{C}$ for $20 \mathrm{~min}$, followed by three washes of PBS. Subsequently, DNA was denatured by incubation of $2 \mathrm{M} \mathrm{HCl}$ at $37^{\circ} \mathrm{C}$ for $60 \mathrm{~min}$, followed by three washes in borate buffer ( $0.1 \mathrm{M}$ borate buffer, $\mathrm{pH} 8.5)$. After incubation with the blocking buffer, cells were stained with anti-BrdU antibody (BD Biosciences, 1:100) overnight at $4^{\circ} \mathrm{C}$. After washing in PBS, cells were incubated with Texas Red-conjugated anti-mouse goat $\mathrm{IgG}$ for $30 \mathrm{~min}$ at RT. After washing, cells were mounted and BrdU-positive cells were manually scored under an immunofluorescence microscope.

Mitotic events were scored by time-lapse videomicroscopy and DNA staining. Cells were synchronized as described above. Real-time images were captured every 10 min with the Openlab software. Mitotic events of control and C53-depleted cells were scored by their morphological change (from flat to rounded). For each experiment, at least 
A

\begin{tabular}{llcl}
\multicolumn{1}{c}{ Bait } & Prey & Growth & $\beta$-gal \\
\hline & & & \\
pGBKT7 & pACT2 & - & - \\
pGBKT7 & pACT2-C53 & - & - \\
pGBKT7-preS2 LHBs & pACT2-C53 & + & + \\
pGBKT7-preS2 LHBs & pACT2 & - & - \\
pGBKT7-lamin C & pACT2-C53 & - & -
\end{tabular}

C

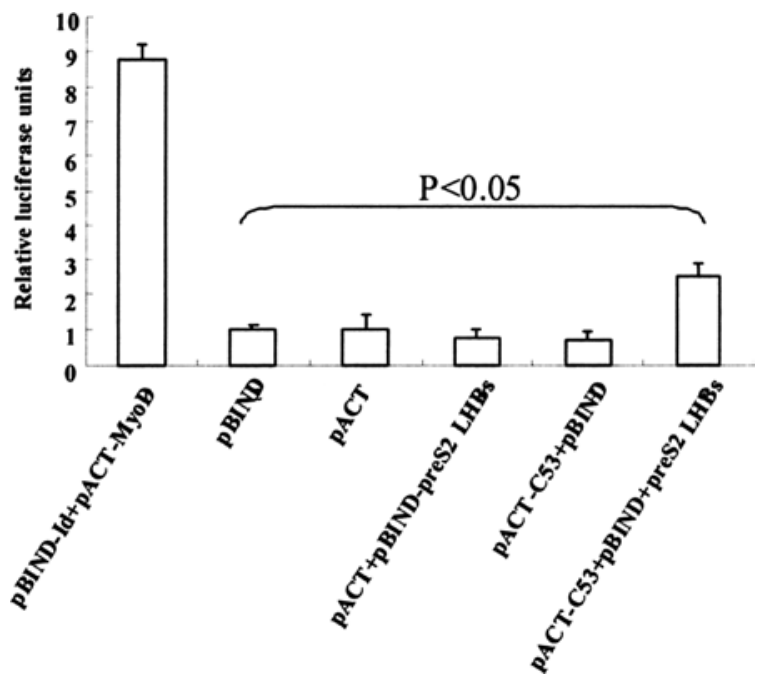

$\mathbf{E}$
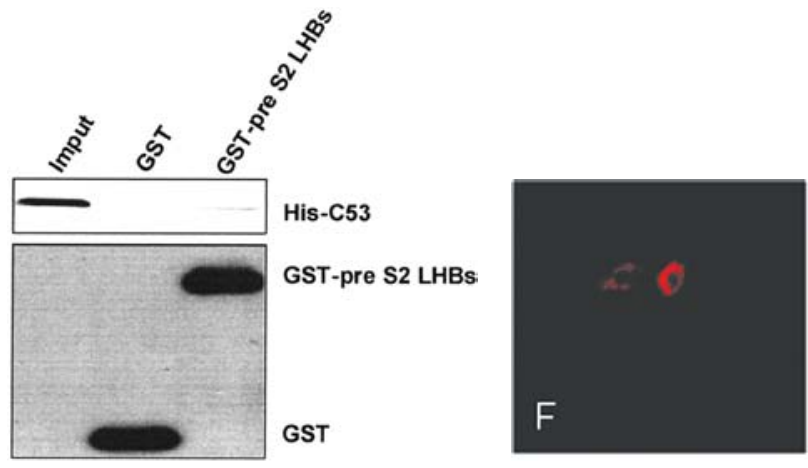

B Myc

Myc-pre S2 LHBs
Flag-C53
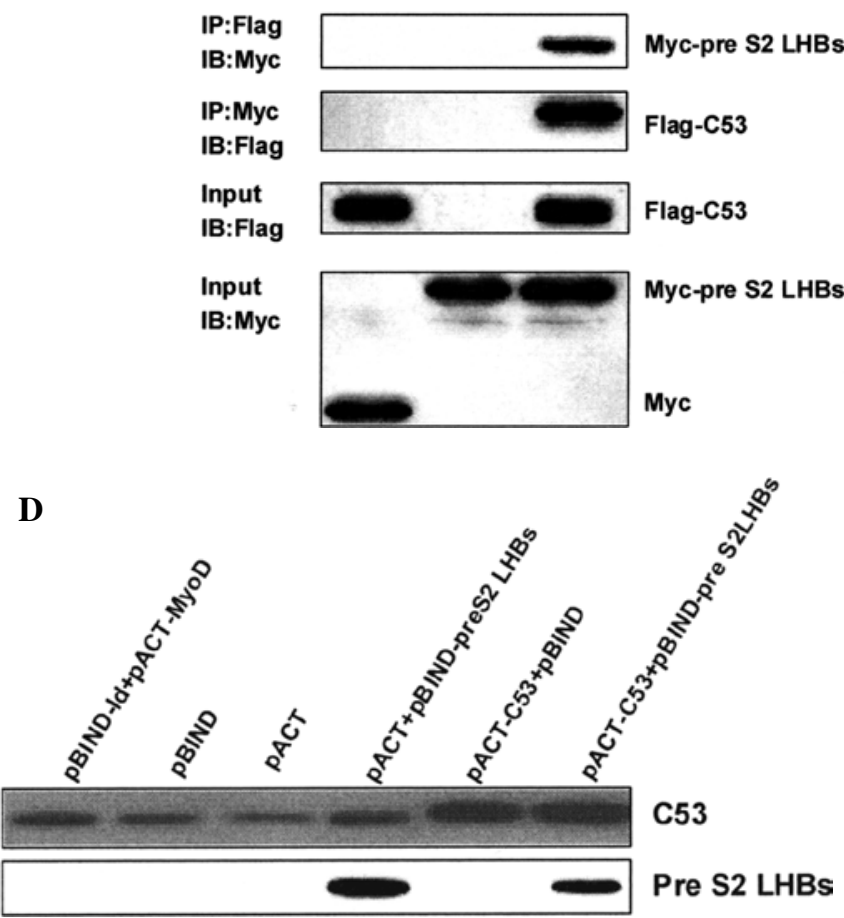

Figure 1. C53 interacts with pre-S2 LHBs in yeast, in mammalian cells and in vitro. (A) Identification of C53 as a pre-S2 LHBs-interacting protein by the yeast two-hybrid assay. Yeast AH109 cells were transformed with different plasmids and grown on SD/-Trp-Leu-His-Ade. +, grown within 96 h; -, no growth within 96 h. Positive colonies were tested for $\beta$-galactosidase activity. +, turned blue within $2 \mathrm{~h}$; -, did not turn blue within $2 \mathrm{~h}$. (B) Interaction of C53 with pre-S2 LHBs in mammalian cells. L02 cells, cultured in regular medium, were transfected with expression plasmids as indicated. Immunoprecipitation (IP) and immunoblotting (IB) was performed using the anti-FLAG or anti-Myc monoclonal antibodies (Santa Cruz Biotechnology), respectively. (C) Assessment of C53 interaction with pre-S2 LHBS in vivo by the mammalian two-hybrid system. L02 cells were transfected with expression plasmids as indicated and cultured in regular medium. At $48 \mathrm{~h}$ after transfection, cells were evaluated by firely luciferase assays. Significance was determined on the mean of three different experiments. The luciferase levels of pACT-C53 and pBIND-pre-S2 LHBS were at least 2.54-fold higher than in the negative control (P<0.05). These results indicate that C53 interacted with pre-S2 LHBs in vivo and in vitro. (D) Expression of C53 and pre-S2 LHBs in the lysate of those cells. The lysates of L02 cells transfected with those plasmids were detected by IB with anti-C53 and anti-LHBs monoclonal antibody. (E) Interaction of pre-S2 LHBs with C53 in vitro. Glutathione-sepharose beads bound with FLAG-pre-S2 LHBs or with FLAG were incubated with His-C53. After washing the beads, the bound proteins were eluted and subjected to SDS-PAGE and autoradiography. To demonstrate the co-localization of C53 and pre-S2 LHBs in L02 cells. Cells were cultured then co-transfected with plasmids. After $48 \mathrm{~h}$, cells were treated with PFA and DAPI, and confocal images were acquired using the Zeiss 510 META confocal microscope. (F) C53 protein (red) localized in the cytoplasm. (G) Pre-S2 LHBs (green) localized in the endoplasmic reticulum. (H) The nuclei of cells (blue) were stained by DAPI. (I) The overlaid images indicate that C53 overlapped partly with pre-S2 LHBs on the surface of the endoplasmic reticulum.

800 cells of control or C53-depleted cells were videotaped,tracked and analyzed. Alternatively, nocodazole (100 ng/ $\mathrm{ml}$ ) was added into the medium after release, cells were collected, fixed and stained with DNA dye (Hoechst 33258). Mitotic cells were scored by nuclear morphology and DNA condensation. Alternatively, the mitotic index was evaluated by phospho-H3 staining and flow cytometric analysis.
In vitro kinase assay. The in vitro Cdk1 kinase assay was performed as described in Jiang et al (17) with minor modification. Cdk1 was immunoprecipitated with Cdk1 antibody (Cell Signaling).

Statistical analysis. Significance was determined using the one-way ANOVA test on the mean of three different experiments. Significance was determined using the paired Student's 
A

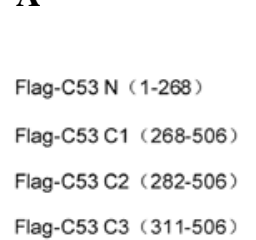

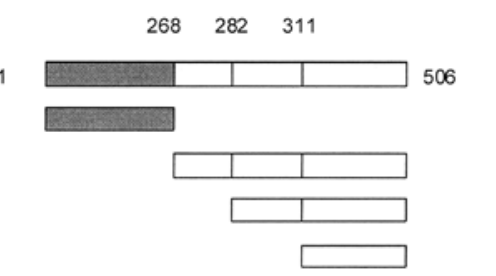

B

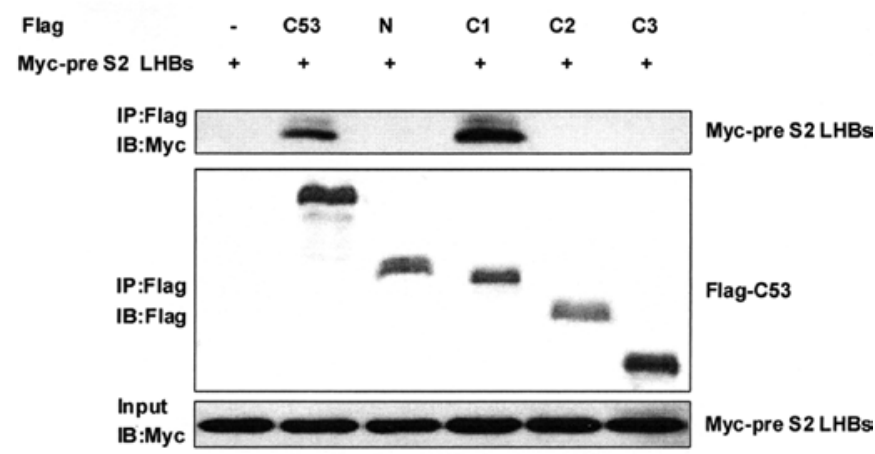

Figure 2.Mapping of the C53 and pre-S2LHBs interaction regions.(A) Mapping of pre-S2 LHBs interaction region in C53. (B) Co-immunoprecipitation of C53 and pre-S2 LHBs. Mapping of C53's pre-S2 LHBs-interacting domains. Myc-pre-S2 LHBs and C53-Flag and its derivatives were overexpressed in L02 cells. C53-Flag protein was pulled down by Protein G Plus/Protein A agarose suspension beads. The presence of pre-S2 LHBs was detected by Myc immunoblotting.

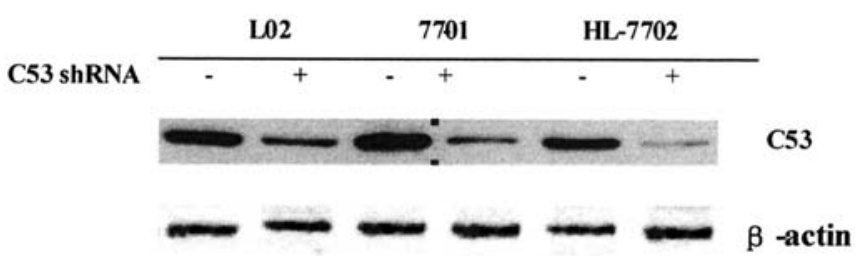

Figure 3. Western blot analysis for the expression of LHBs protein. The results showed that expressions of C53 in cells and C53 shRNA expression vectors pAdTrack-CMVC53, respectively.

t-test on the mean of three different experiments. Probabilities of 0.05 or less were considered to be statistically significant.

\section{Results}

Identification of C53 as a pre-S2 LHBs-interacting protein by a yeast two-hybrid system. To identify novel pre-S2 LHBsinteracting proteins, we performed a yeast two-hybrid screening of a human liver cDNA library using full-length pre-S2 LHBs as bait. Screening of 4 million transformants resulted in the isolation of several positive clones, which encoded the full-length cDNA of the C53 gene. To further confirm the interaction of C53 with pre-S2 LHBs in yeast, we next examined the ability of C53 protein to bind to pre-S2 LHBs in yeast cells (Fig. 1A). These data demonstrate that C53 interacts with pre-S2 LHBs in yeast cells.

Interaction of C53 with pre-S2 LHBs in vivo and in vitro. To confirm the interaction between C53 and pre-S2 LHBs, we next examined the ability of C53 protein to bind to pre-S2 LHBs in mammalian cells. Cells were co-transfected with Flag-tagged C53, Myc-tagged pre-S2 LHBs, or Myc control vector. Immunoprecipitation (IP) of cell lysates with an antiFlag monoclonal antibody was followed by immunoblotting (IB) with anti-Myc. Results showed a specific interaction between the Flag-C53 and Myc-pre-S2 LHBs (Fig. 1B). To test the hypothesis of C53 interacting with pre-S2 LHBs in vivo, the CheckMate mammalian two-hybrid system (Promega) was used. The plasmids pACT-C53 and pBIND- pre-S2 LHBs were used for co-transfections with Lipofectamine 2000. The plasmids pACT-C53+pBIND, pACT+pBIND-pre-S2 LHBs, pACT, pBIND and blank were used for transfections, respectively, as a negative control. The plasmids pBIND-Id and pACT-MyoD Control Vector were co-transfected as a positive control. After $48 \mathrm{~h}$ transfection, cells were lysed. Luciferase assays were performed using the Dual-Glo Luciferase Assay System (Promega) following the manufacturer's instructions. The results were corrected for transfection efficiency using Renilla luciferase. Significance was determined using the paired Student's t-test on the mean of three different experiments. The luciferase levels of pACT-C53 and pBIND-pre-S2 LHBs were at least 2.54-fold higher than in the negative control $(\mathrm{P}<0.05)$ (Fig. 1C).

Expression of C53 and pre-S2 LHBs in lysate of those cells was detected by WB with anti-C53 and anti-LHBs monoclonal antibody (Fig. 1D). To demonstrate the interaction of C53 and pre-S2 LHBs in vitro, GST pull-down assays were performed in which in vitro translated His-C53 was incubated with fulllength GST-pre-S2 LHBs or Flag. As shown in Fig. 1E, C53 interacted with GST-pre-S2 LHBs but not with Flag alone. To test the co-localization of C53 and pre-S2 LHBs, cells were grown on glass coverslips in culture plates then co-transfected with plasmids pEGFP-C1-pre-S2 LHBs and pDS-RED1N1-C53. After $48 \mathrm{~h}$, cells were deal with PFA and DAPI, confocal images were acquired using the Zeiss 510 META confocal microscope. C53 protein (red) localized to the cytoplasm (Fig. 1F). Pre-S2 LHBs (green) localized in the endoplasmic reticulum (Fig. 1G). The nuclei of cells (blue) were stained by DAPI (Fig. 1H). The overlaid images indicated that C53 overlapped partly with pre-S2 LHBs on the surface of the endoplasmic reticulum. These results indicate that C53 interacted with pre-S2 LHBs in vivo and in vitro.

Mapping of the pre-S2 LHBs and C53 interaction regions. To identify the region of C53 required for its interaction with pre-S2 LHBs, co-immunoprecipitation experiments were performed in which four deletion mutants were identified. C53 is cleaved by caspase-3 at three sites (D268, D286 and D311, Fig. 2A). As shown in Fig. 2A, both full-length and the carboxy-terminal fragments $\mathrm{C} 53 \mathrm{C} 1$ interacted with Myc-pre-S2 LHBs, but the amino-terminal fragment of C53 (C53N, residues 1-256) did not (Fig. 2A). As shown in Fig. 2B, the Flag-C53 C1 (268-506) bound specifically to pre-S2 LHBs, but the Flag-N (1-268), Flag-C53 C2 (282-506), Flag-C53 C1 (311-506) and Flag did not, suggesting that the 268-282 region is required for the interaction with pre-S2 LHBs. Among the C-terminal cleavage fragments, C53-C1 appeared to bear the highest affinity to Myc-pre-S2 LHBs, indicating that the 
A

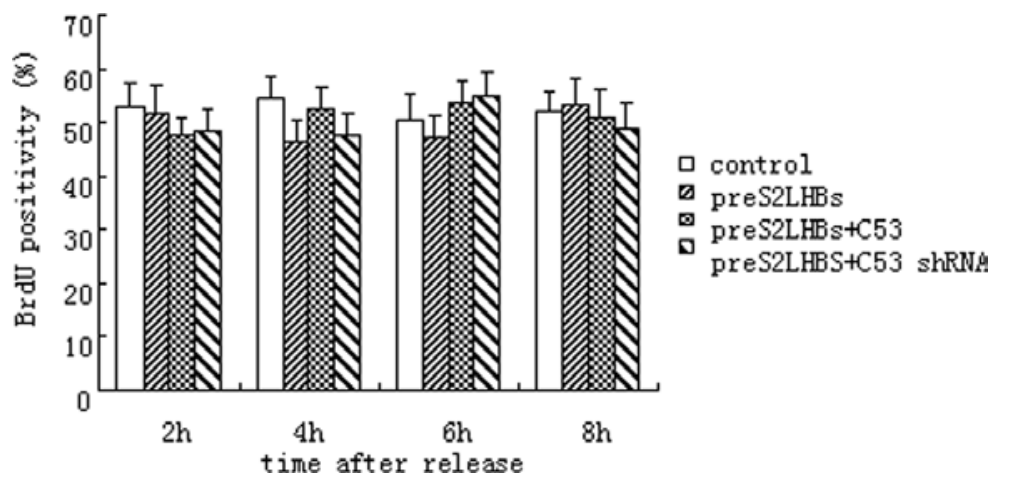

B

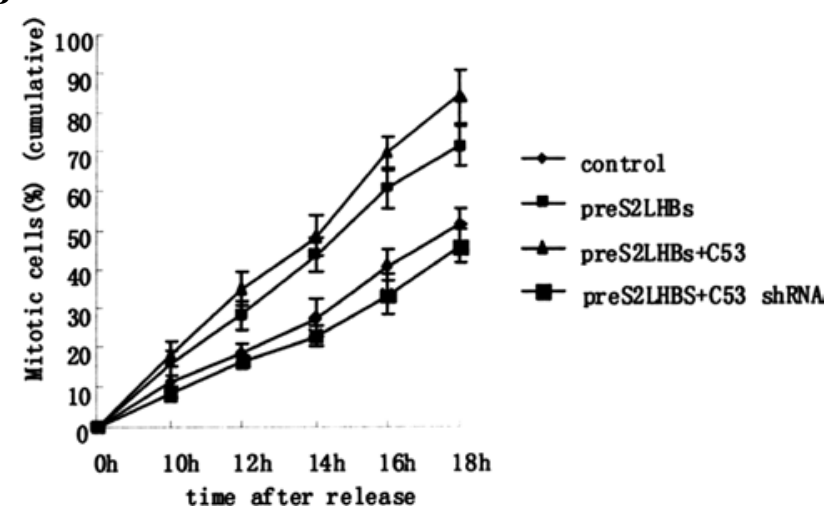

D

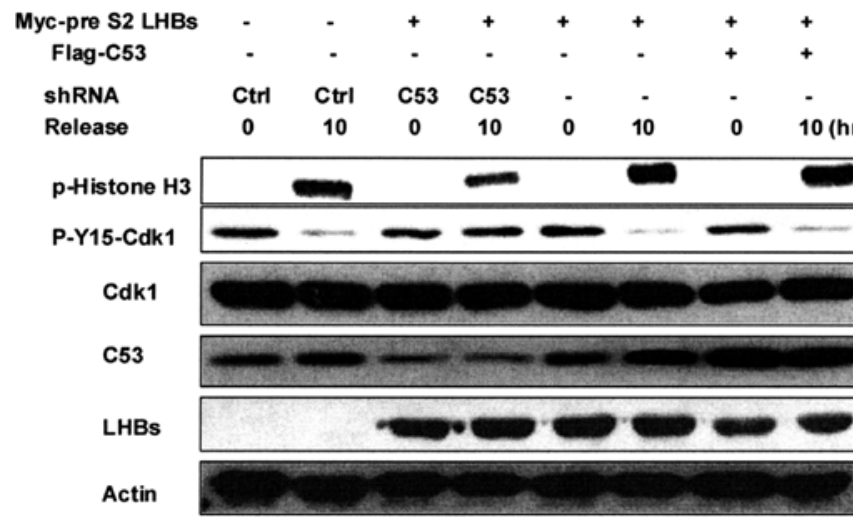

C

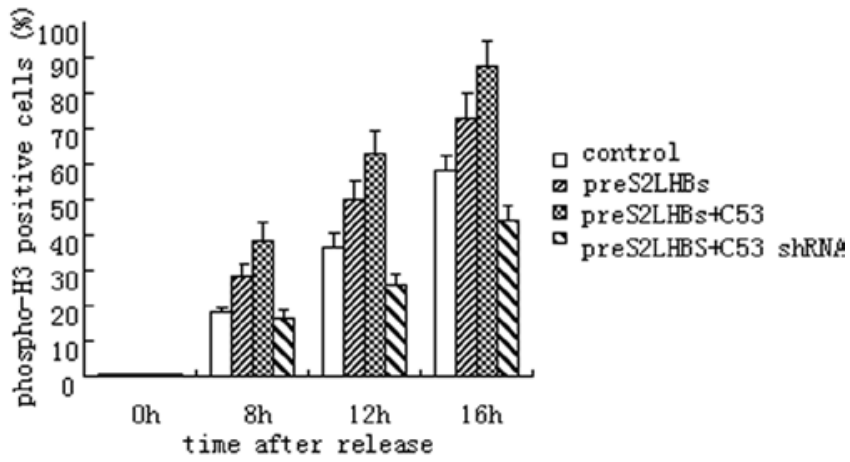

$\mathbf{E}$

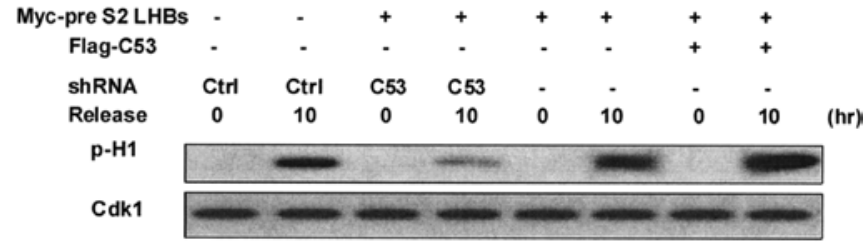

Figure 4. Interaction of C53 with pre-S2 LHBs increases mitotic entry and Cdk1 activation. (A) Interaction of C53 with pre-S2 LHBs did not affect DNA synthesis. L02 cells were transfected with plasmids and synchronized at the G1/S transition as described in Materials and methods. Cells were pulse labeled with $\mathrm{BrdU}(50 \mu \mathrm{M})$ for $30 \mathrm{~min}$ at indicated time points after release from the second thymidine block. BrdU-positive cells were detected by immunostaining and scored manually. More than 500 cells were counted in each of three independent experiments. (B) Interaction of C53 with pre-S2 LHBs promoted mitotic entry. Cell cycle progression of $>1,000$ cells was recorded by time-lapse videomicroscopy. The number of mitotic cells was scored by examination of individual cells. (C) Interaction of C53 with pre-S2 LHBs promoted mitotic entry. Plasmids were transfected into L02 cells by HiPerfect transfection agent. Cells were harvested at indicated time points and subjected to phospho-H3 staining and flow cytometry analysis. (D) Interaction of C53 with pre-S2 LHBs causes less inactive Cdk1. Cell lysates were collected at indicated time points after release from the second block, and subjected to immunoblotting using indicated antibodies. (E) Interaction of C53 with pre-S2 LHBs promoted Cdk1 activation in vitro. Cdk1 was precipitated from cell lysates at the indicated time, and subjected to an in vitro kinase assay with histone H1 as a substrate. Relative intensity was measured with Openlab software and marked at the bottom of the gel. All data are represented as mean \pm SEM.

region between residues 268-282 may be important for C53-pre-S2 LHBs interaction (Fig. 2B). We also examined the pre-S2 LHBs-C53 interaction in vitro using purified C53 proteins which were in agreement with our Co-IP results.
Interaction of C53 with pre-S2 LHBs promotes mitotic entry and Cdk1 activation. Expression of $\mathrm{C} 53$ protein could be inhibited by C53 shRNA in L02, OSG-7701 and HL-7702 cells (Fig. 3). Cells were synchronized at the G1/S boundary by double thymidine block, and then released into mitosis. After 


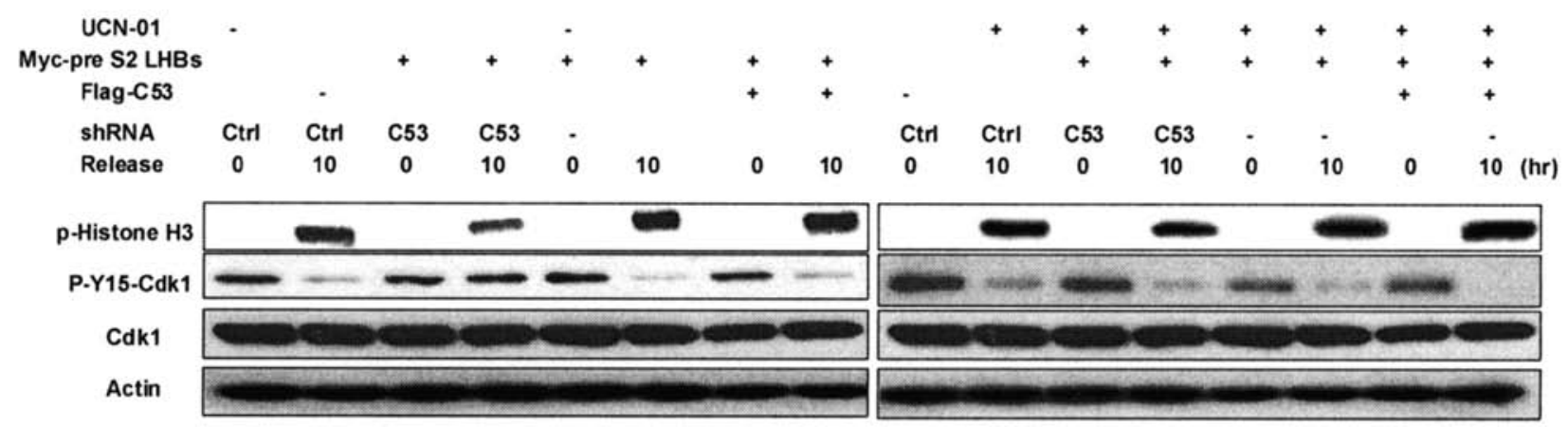

Figure 5. C53 interaction with pre-S2 LHBs delays Chk1 activity. L02 cells were transiently transfected with pre-S2 LHBs and C53 constructs. Cells were collected at $24 \mathrm{~h}$ after transfection. Total cell lysates were subjected to immunoblotting using the indicated antibodies. The Chk1 inhibitor UCN-01 prevented delayed Cdk1 activation. L02 cells were synchronized by a double thymidine block. UCN-01 ( $300 \mathrm{nM})$ was added into the medium at $5 \mathrm{~h}$ after release from the second block. Cells were collected $10 \mathrm{~h}$ after release, and the total cell lysates were subjected to SDS-PAGE and immunoblotting using the indicated antibodies.

$24 \mathrm{~h}$, BrdU was added into the medium at indicated time points to evaluate DNA synthesis. As shown in Fig. 4A, incorporation of BrdU into the control, pre-S2 LHBs transfected, C53 and pre-S2 LHBs transfected, C53 shRNA and pre-S2 LHBs transfected cells, did not significantly vary between the groups (Fig. 4A), indicating that interaction of C53 with pre-S2 LHBs may not affect the G1/S transition and DNA synthesis. In contrast, accumulation of mitotic cells was significantly promoted in cells transfected with pre-S2 LHBs+C53 or pre-S2 LHBs, and delayed in C53-knockdown cells transfected with pre-S2 LHBs (Fig. 4B). To further examine the specific effect of the interaction of C53 with LHBs on mitotic entry, we repeated this experiment and evaluated the mitotic entry using phospho-H3 staining and flow cytometry. As shown in Fig. 4C, pre-S2 LHBs+C53 or pre-S2 LHBs significantly promoted mitotic entry and pre-S2 LHBs+C53 shRNA significantly delayed mitotic entry as noted by changes in the percent of phospho-H3 positive cells. Promotion of mitosis was further confirmed by our observation that there was increased phosphorylation of histone $\mathrm{H} 3$ in pre-S2 LHBs+C53 cells than in the control cells and less in pre-S2 LHBs+C53 shRNA (Fig. 4D, panel of p-histone H3). We also examined whether pre-S2 LHBs+C53 promotes Cdk1 activation and pre-S2 LHBs+C53 shRNA delays Cdk1 activation. Consistently, pre-S2 LHBs+C53 or pre-S2 LHBs-transefcted cells contained more actived Cdk1 than control cells, and pre-S2 LHBs+C53 shRNA cells contained more inactive Cdk1 than the control cells (Fig. 4D, panels of Cdk1 and p-Y15-Cdk1) $(\mathrm{P}<0.05)$. Moreover, an in vitro kinase assay showed that $\mathrm{Cdk} 1$ in pre-S2 LHBs+C53 cells was more active and pre-S2 LHBs+C53 shRNA cells was less active (Fig. 4E). Together, our data strongly indicate that the interaction of pre-S2 LHBs with C53 may serve as a positive regulator for Cdk1 activation at the onset of mitosis.

Interaction of C53 with pre-S2 LHBs delays Chkl activation. If indeed the interaction of pre-S2 LHBs with C53 negatively regulates Chk1 activity, we speculated that the role of the pre-S2 LHBs interaction with C53 may be the same as the role of Chk1 inhibition which reverses delayed Cdk1 activation induced by C53 knockdown. The Chk1 inhibitor UCN-01 (7-hydroxystaurosporine) (300 $\mathrm{nM})$ was added $5 \mathrm{~h}$ after the cells were released from the second thymidine block. As shown in Fig. 4, addition of UCN-01 abolished delayed Cdk1 activation caused by C53 knockdown. Meanwhile, the role of pre-S2 LHBs interacted with C53 was the same as UCN-01. This result indicates that the interaction of pre-S2 LHBs with C53 delays Chk1 activity, further promoting Cdk1 activation and the onset of mitosis (Fig. 5).

\section{Discussion}

Some studies led to the identification of a topogenic element mapped to a pre-S1 C-terminal sequence located between residues 70 and 94, termed cytosolic anchorage determinant (CAD). CAD interacts with the cognate heat-shock protein Hsc-70 preventing pre-S co-translational translocation into an e-pre-S topology and glycosylation of the Asn-15 and Asn-4 of the pre-S1 and pre-S2 domains, respectively $(20,21)$. When anchored on the ER, the entire pre-S domain of the large envelope protein is exposed in the cytosol.

Interestingly, our findings suggest that the interaction between pre-S2 LHBs and C53 by yeast two-hybrid system, mammalian two-hybrid analysis, co-immunoprecipitation, GST-pull down and co-localization. We show that pre-S2 LHBs physically interacts with C53 in vitro and in vivo, but not with LHBs or other mutants. The overlaid images of confocal microscopy indicated that C53 overlapped partly with pre-S2 LHBs on the surface of the endoplasmic reticulum (Fig. 1). This result suggests that the CAD of pre-S2 LHBs may interact with C53.

Jiang et al have reported that C53 protein is a novel regulator of checkpoint kinases in cell cycle control (16). Their study demonstrated that C53 antagonizes the checkpoint kinases increasing Cdk1 activation, and C53 depletion delays Cdk1 activation and mitotic entry in unperturbed cell cycle progression. Moreover, C53 overexpression partially inhibits checkpoint kinase activation in DNA damage response, thereby overriding the $\mathrm{G} 2 / \mathrm{M}$ checkpoint-mediated Cdk1 inactivation. Intriguingly, they found that C53 interacts with Chk1 and counteracts its activity to promote Cdk1 activation both locally and globally. Taken together, their results strongly suggest that $\mathrm{C} 53$ acts as a promoter for Cdk1 activation through antagonizing checkpoint kinases (16).

Our findings demonstrated that incorporation of BrdU into the control, pre-S2 LHBs transfected, C53 and pre-S2 LHBs transfected, C53 shRNA and pre-S2 LHBs-transfected 
cells did not significantly differ during cell cycle progression (Fig. 4A), and the interaction of C53 with pre-S2 LHBs may not affect the G1/S transition and DNA synthesis. In contrast, accumulation of mitotic cells was significantly promoted in cells transfected with pre-S2 LHBs+C53 or pre-S2 LHBs, and delayed in C53-knockdown cells transfected with pre-S2 LHBs (Fig. 4B). As shown in Fig. 4C, pre-S2 LHBs+C53 or pre-S2 LHBs significantly promoted mitotic entry and LHBs+C53 shRNA significantly delayed mitotic entry using phospho-H3 staining and flow cytometry. Mitotic promotion was further confirmed by our observation that there was increased phosphorylation of histone $\mathrm{H} 3$ in LHBs+C53 cells than in the control cells and less in pre-S2 LHBs+C53 shRNA (Fig. 4D). We also examined whether pre-S2 LHBs+C53 promotes Cdk1 activation and pre-S2 LHBs+C53 shRNA delays Cdk1 activation. Consistently, pre-S2 LHBs+C53 or pre-S2 LHBs transefcted cells contained more actived Cdk1 than control cells, and pre-S2 LHBs+C53 shRNA cells contained more inactive Cdk1 than the control cells (Fig. 4D). Moreover, an in vitro kinase assay showed that Cdk1 in pre-S2 LHBs+C53 cells was more active and pre-S2 LHBs+C53 shRNA cells was less active (Fig. 4E). Together, our data strongly indicate that the interaction of pre-S2 LHBs with C53 may serve as a positive regulator for Cdk1 activation at the onset of mitosis.

Cells utilize the checkpoint mechanisms to coordinate cell cycle progression and cellular response to cell cycle irregularities, such as replication stress and DNA damage, thereby maintaining accurate transmission of genetic material during cell division. One of the key checkpoints is the G2/M checkpoint that regulates activation of Cdk1 (cyclin dependent kinase 1) and mitotic entry. In an unperturbed cell cycle, the components of the checkpoint are also essential for surveillance of cell cycle progression. For example, Chk1 is essential for animal development and proper cell cycle progression (22-24). Inhibition of Chk1-mediated signaling may lead to increased DNA replication and DNA breakage, aberrant mitotic entry and apoptosis $(22,25)$.

Chk1 activity has been reported to be responsible for delayed Cdk1 activation in C53 depleted cells at the onset of mitosis, further supporting the notion that C53 is an antagonist of Chk1, which is essential for unperturbed cell cycle progression. The results suggest that C53 may directly inhibit Chk1 kinase activity in vivo through an activity localized at its $\mathrm{C}$-terminal fragment; it is likely that C53 exerts its function through modulating Chk1 activity in an unperturbed cell cycle (16).

Our study supports the notion that the interaction of pre-S2 LHBs with C53 delays Chk1 activity, further promoting Cdk1 activation and the onset of mitosis (Fig. 5). The role of pre-S2 LHBs interaction with C53 may be the same as the role of Chk1 inhibition which reverses delayed Cdk1 activation induced by C53 knockdown. The addition of UCN-01 abolished delayed Cdk1 activation caused by C53 knockdown. The funtion of the pre-S2 LHBs interaction with $\mathrm{C} 53$ was similar to UCN-01.

Recent studies strongly suggest that cell cycle checkpoints are essential for maintenance of replication accuracy and genome stability, and defects in the checkpoints are largely responsible for tumorigenesis and genome instability of cancer cells (26). Many efforts have been made to identify checkpoint kinase inhibitors as chemo- and radio-sensitizing agents in cancer treatment (27).Our study strongly suggests that the binding of pre-S2 LHBs with C53 is a novel negative regulator of the checkpoint response. By counteracting C53, pre-S2 LHBs promotes Cdk1 activation and mitotic entry in unperturbed cell cycle progression and delays the function of Chk1, which may be a novel potential mechanism for HBV-induced hepatocellular carcinoma (HCC). Identification of the interaction of C53 with pre-S2 LHBs as a negative regulator of checkpoint kinases sheds light on a novel regulatory mechanism for checkpoint response, and provides a basis for development of novel chemosensitizing agents for the treatment on $\mathrm{HCC}$ related to HBV.

\section{Acknowledgements}

This work was supported by a grant from the National Natural Science Foundation of China (nos. 30600524 and 30870991).

\section{References}

1. Hollinger FB and Liang TJ: Hepatitis B virus. In: Fields Virology. Knipe DM and Howley PM (eds). Lippincott-Raven, Philadelphia, pp2971-3036, 2001.

2. Lee WM: Hepatitis B virus infection. N Engl J Med 337: 1733-1745, 1997.

3. Beasley RP: Hepatitis B virus. The major etiology of hepatocellular carcinoma. Cancer 61: 1942-1956, 1988.

4. McMahon BJ: Hepatocellular carcinoma and viral hepatitis. In: Viral Hepatitis: Diagnosis, Treatment, Prevention. Wilson RA (ed). Marcel Dekker, New York, pp315-330, 1997.

5. Chan HL and Sung JJ: Hepatocellular carcinoma and hepatitis B virus. Semin Liver Dis 26: 153-161, 2006.

6. Seeger C and Mason WS: Hepatitis B virus biology. Microbiol Mol Biol Rev 64: 51-68, 2000.

7. Heermann KH, Goldmann U, Schwartz W, Seyffarth T, Baumgarten $\mathrm{H}$ and Gerlich WH: Large surface proteins of hepatitis B virus containing the pre-s sequence. J Virol 52: 396-402, 1984.

8. Nassal M: Hepatitis B virus morphogenesis. Curr Top Microbiol Immunol 214: 297-337, 1996.

9. Fan YF, Lu CC, Chang YC, Chang TT, Lin PW, Lei HY and Su IJ: Identification of a pre-S2 mutant in hepatocytes expressing a novel marginal pattern of surface antigen in advanced disease of chronic hepatitis B virus infection. J Gastroenterol Hepatol 15: $519-528,2000$.

10. Fan TF, Lu CC, Chen WC, Yao WJ, Wang HC, Chang TT, Lei HY, Shiau Al and Su IJ: Prevalence and significance of hepatitis B virus (HBV) pre-S mutants in serum and liver at different replicative stages of chronic HBS infection. Hepatology 33: 277-286, 2001.

11. Fang ZL, Sabin CA, Dong BQ, Wei SC, Chen QY, Fang KX, Yang JY, Huang J, Wang XY and Harrison TJ: Hepatitis B virus pre-S deletion mutations are a risk factor for hepatocellular carcinoma: a matched nested case-control study. J Gen Virol 89: 2882-2890, 2008.

12. SuwannakarnK, Tangkijvanich $P$, ThawornsukN, Theamboonlers A Tharmaphornpilas P, Yoocharoen $\mathrm{P}$, Chongsrisawat V and Poovorawan Y: Molecular epidemiological study of hepatitis B virus in Thailand based on the analysis of pre-S and $\mathrm{S}$ genes. Hepatol Res 38: 244-251, 2008.

13. Wang HC, Wu HC, Chen CF, Lei HY and Su IJ: Different types of ground glass hepatocytes in chronic hepatitis B virus infection contain specific pre-S mutants that may induce endoplasmic reticulum stress. Am J Pathol 163: 2441-2449, 2003.

14. Hsieh YH, Su IJ, Wang HC, Chang WW, Lei HY, Lai MD, Chang WT and Huang W: The pre-S mutant surface antigens in chronic hepatitis B virus infection induce oxidative stress and DNA damage. Carcinogenesis 25: 2023-2032, 2004.

15. Wang HC, Chang WT, Chang WW, Wu HC, Huang W, Lei HY, Lai MD, Fausto $\mathrm{N}$ and Su IJ: Upregulation of cyclin A and nodular proliferation of hepatocytes induced by a pre-S2 deletion mutant in chronic HBV infection. Hepatology 41: 761-770, 2005 
16. Jiang $\mathrm{H}, \mathrm{Wu} \mathrm{J}, \mathrm{He} \mathrm{C}$, Yang $\mathrm{W}$ and $\mathrm{Li} \mathrm{H}$ : A tumor suppressor C53 protein antagonizes checkpoint kinases to promote cyclindependent kinase 1 activation. Cell Res 19: 458-468, 2009.

17. Jiang $\mathrm{H}$, Luo $\mathrm{S}$ and $\mathrm{Li} \mathrm{H}$ : Cdk5 activator-binding protein $\mathrm{C} 53$ regulates apoptosis induced by genotoxic stress via modulating the G2/M DNA damage checkpoint. J Biol Chem 280: 20651-20659, 2005.

18. Li H, Bergeron L, Cryns V, Pasternack MS, Zhu H, Shi L, Greenberg A and Yuan J: Activation of caspase-2 in apoptosis. J Biol Chem 272: 21010-21017, 1997.

19. Hirota T, Kunitoku N, Sasayama T, Marumoto T, Zhang D, Nitta M, Hatakeyama K and Saya H: Aurora-A and an interacting activator, the LIM protein Ajuba, are required for mitotic commitment in human cells. Cell 114: 585-598, 2003.

20. Eddleston A: Modern vaccines. Lancet 335: 1142-1145, 1990

21. Fernholz D, Stemler M, Brunetto M, Bonino F and Will H: Replicating and virion secreting hepatitis B mutant virus unable to produce preS2 protein. J Hepatol 13: S102-S104, 1991.

22. Liu Q, Guntuku S, Cui XS, Matsuoka S, Cortez D, Tamai K, Luo G, Carattini-Rivera S, DeMayo F, Bradley A, Donehower LA and Elledge SJ: Chk1 is an essential kinase that is regulated by Atr and required for the G(2)/M DNA damage checkpoint. Genes Dev 14: 1448-1459, 2000.
23. Takai H, Tominaga K, Motoyama N, Minamishima YA, Nagahama H, Tsukiyama T, Ikeda K, Nakayama K, Nakanishi M and Nakayama K: Aberrant cell cycle checkpoint function and early embryonic death in Chk1(-/-) mice. Genes Dev 14: 1439-1447, 2000.

24. Brown EJ and Baltimore D: ATR disruption leads to chromosomal fragmentation and early embryonic lethality. Genes Dev 14: 397-402, 2000

25. Syljuasen RG, Sorensen CS, Hansen LT, Fugger K, Lundin C, Johansson F, Helleday T, Sehested M, Lukas J and Bartek J: Inhibition of human Chk1 causes increased initiation of DNA replication, phosphorylation of ATR targets, and DNA breakage. Mol Cell Biol 25: 3553-3562, 2005.

26. Nojima H: G1 and S-phase checkpoints, chromosome instability, and cancer. Methods Mol Biol 280: 43-49, 2004.

27. Zhou BB and Bartek J: Targeting the checkpoint kinases: chemosensitization versus chemoprotection. Nat Rev Cancer 4: 216-225, 2004 